LiTH-ISY-R-1991

\title{
Moving the State Between Equilibria
}

\author{
Mats Jirstrand and Torkel Glad \\ Department of Electrical Engineering \\ Linköping University, S-581 83 Linköping, Sweden \\ www: http://www. control.isy.liu.se \\ email: $\{$ matsj,torkel\}@isy.liu.se
}

1997-11-14

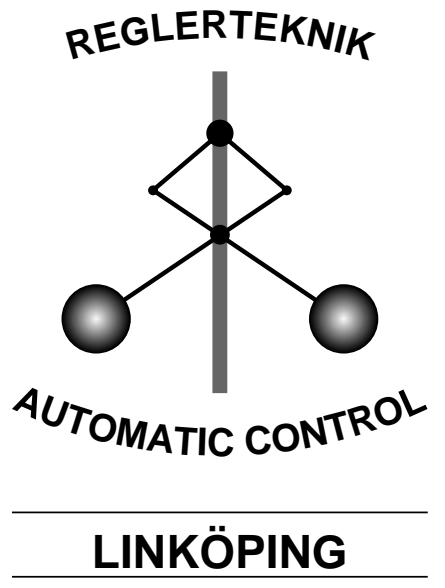

Technical reports from the Automatic Control group in Linköping are available as UNIX-

compressed Postscript files by anonymous ftp at the address ftp.control.isy.liu.se. 


\title{
Moving the State Between Equilibria
}

\author{
M. Jirstrand and T. Glad \\ Department of Electrical Engineering \\ Linköping University, 58183 Linköping, Sweden \\ Email: \{matsj,torkel\}@isy.liu.se \\ WWW: www.control.isy.liu.se
}

\begin{abstract}
We consider the problem of moving the system state between different equilibrium points. For systems whose linearizations are exponentially stable along a curve connecting the given points we show that the transfer can be achieved in finite time. The result can be extended to systems whose linearizations are stabilizable.
\end{abstract}

\section{Introduction}

Consider a nonlinear system of the form $\dot{x}=f(x, u)$. An equilibrium point (stationary point) corresponding to a constant control $u_{0}$ is a point $x_{0}$ such that $f\left(x_{0}, u_{0}\right)=0$. In the process industry the controlled system is often operated at several different equilibrium points, corresponding to different set-points. It is then necessary to transfer the system state from one equilibrium point to another. The purpose of this paper is to investigate conditions under which it is possible to do this. We will concentrate on the situation where two equilibrium points in the $x$ - $u$-space are connected via a curve consisting of equilibrium points.

Some problems related to ours have been treated in the literature. In [2] a single input nonlinear system is uniformly approximated such that the systems agree to first order on the set of equilibrium points and the approximate system is input-to-state linearizable in an neighborhood of the equilibrium points. Then a control law for the feedback linearized system is used to control the system in a neighborhood of the equilibrium points.

A time-varying linear quadratic controller is derived in [5] for changing the set-point of a nonlinear system. 
In [8] a robust controller design method is proposed. The system is linearized around all stationary points and a robust controller is designed for each such system. The set-point can then be changed by switching among the controllers.

Here follow some notations and definitions we use. A matrix $A$ is called Hurwitz if all its eigenvalues belong strictly to the left half plane. A positive (semi-) definite matrix is denoted $P \succ 0,(P \succeq 0)$. The functional matrix of $f(x, u)$ w.r.t. $x$ is denoted $f_{x}(x, u) . B_{r}\left(x_{c}\right)$ is a ball of radius $r$, centered at $x_{c}$ of appropriate dimension and $\operatorname{dist}(x, y)$ is the Euclidean distance function.

\section{Set-Point Transfer}

Consider the following nonlinear dynamic system

$$
\dot{x}=f(x, u), \quad f: \mathbb{R}^{n} \times \mathbb{R}^{m} \rightarrow \mathbb{R}^{n},
$$

where $f(x, u)$ is a twice continuously differentiable function of the state $x$ and control $u$. The stationary points or equilibria are given by the set

$$
\mathcal{E}=\left\{(x, u) \in \mathbb{R}^{n} \times \mathbb{R}^{m} \mid f(x, u)=0\right\} .
$$

Among these the asymptotically stable points are given by

$$
\mathcal{E}_{a s}=\left\{(x, u) \in \mathbb{R}^{n} \times \mathbb{R}^{m} \mid f(x, u)=0 \wedge f_{x}(x, u) \text { is Hurwitz }\right\} .
$$

The states that can be made stationary by an admissible choice of the control will be called stationarizable points of (1) and are given by the projection of the set $\mathcal{E}$ onto the state space, i.e.,

$$
\mathcal{S}=\left\{x \in \mathbb{R}^{n} \mid \exists u \in \mathbb{R}^{m} \quad f(x, u)=0\right\} .
$$

If $m=1$ we have a curve of stationarizable points and if $m>1$ we get a hyper surface implicitly parameterized by the control $u$. Similarly the set of asymptotically stable stationarizable points of (1) are given by

$$
\mathcal{S}_{a s}=\left\{x \in \mathbb{R}^{n} \mid \exists u \in \mathbb{R}^{m} \quad f(x, u)=0 \wedge f_{x}(x, u) \text { is Hurwitz }\right\} .
$$

The set $\mathcal{S}_{a s}$ is important since it consists of all practical operating points of the system (1), which can be used without a stabilizing controller.

Note that if the dynamics is given by a set of polynomial differential equations then the above sets can be described by multivariate polynomial equalities and inequalities in $x$ and $u$. A survey of computational tools for 
these so called semi-algebraic sets can be found in [3]. In [1] computations of equilibria for nonlinear aircraft dynamics using computer algebra methods is addressed. For systems modeled by polynomial differential equations the results in this paper can be constructively utilized using the tools in the abovementioned papers.

In this paper we will investigate the following problem:

Given two points $x_{a} \in \mathcal{S}_{a s}$ and $x_{b} \in \mathcal{S}_{a s}$. Is it possible to transfer the state from $x_{a}$ to a neighborhood of $x_{b}$ in finite time?

For a linear system, $\dot{x}=A x+B u$, we know that this is possible for any two points on $\mathcal{S}_{a s}$ if $A$ is Hurwitz since the system then is globally asymptotically stable for any constant $u \equiv u_{0}$, which corresponds to the stationary point $x_{0}=-A^{-1} B u_{0}$.

For nonlinear systems the situation is much more intricate. First, it is usually hard to determine global asymptotic stability of the system. Second, $\mathcal{E}$ or $\mathcal{E}_{a s}$ may consist of several non connected components even if $\mathcal{S}$ or $\mathcal{S}_{a s}$ are connected. An example of this is the scalar system

$$
\dot{x}=\left(u^{2}+4 u-x+3\right)\left(u^{2}-4 u+x+3\right)(x-u) .
$$

The set $\mathcal{E}$ consists of two parabolas and one straight line in the $u$ - $x$-space, se Figure 1 . The linearizations along the parabolas are asymptotically stable,

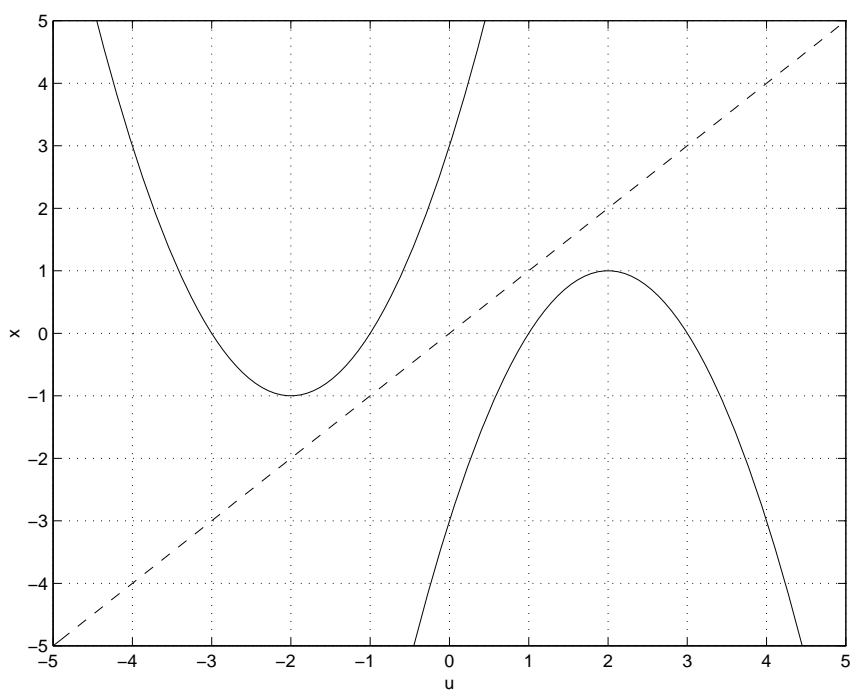

Figure 1: Equilibrium points of (6). Solid lines: asymptotically stable linearizations, dashed lines: unstable linearizations. 
while the linearizations along the straight line are unstable. The set $\mathcal{E}_{a s}$ is thus not connected, while its projection $\mathcal{S}_{a s}$ on the $x$-axis is.

In practise it is often possible to transfer the system state between two asymptotically stable stationary points on the same component by changing the control input slowly between its corresponding initial and final values. This suggests that if we can only keep the state sufficiently close to the set of asymptotically stable stationarizable points during the transfer we will succeed.

In this paper we will show that given a curve $\Gamma \in \mathcal{S}_{\text {as }}$ entirely in one component of $\mathcal{S}_{a s}$, starting at $x_{a}$ and ending at $x_{b}$, it is possible to transfer the state from $x_{a}$ to a neighborhood of $x_{b}$ in a finite amount of time.

To estimate the region of attraction of a curve of asymptotically stable stationarizable points we need the following lemma.

Lemma 1 Let $A_{s}: s \in \Omega \rightarrow \mathbb{R}^{n \times n}$ be a continuous matrix valued function where $\Omega \in \mathbb{R}$ is a closed interval. Furthermore, assume that $A_{s}$ is Hurwitz for all $s \in \Omega$. Then there exist uniform bounds on the solution of the following Lyapunov equation

$$
A_{s}^{T} P_{s}+P_{s} A_{s}=-I, \quad P_{s}^{T}=P_{s},
$$

i.e., there exists constants $\lambda_{m}<\lambda_{M}<\infty$ such that

$$
\lambda_{m} I \preceq P_{s} \preceq \lambda_{M} I, \quad \forall s \in \Omega .
$$

Proof: Since $A_{s}$ is Hurwitz it is well known that that equation (7) has a unique positive definite solution, see [7]. Now, (7) is a system of linear equations and the uniqueness of its solution implies that the corresponding determinant is nonzero. This means that the entries of $P_{s}$ are continuous functions of the entries of $A_{s}$ since the solution of a nonsingular system of linear equations can be expressed as rational functions of the entries of the system matrix and the right hand side vector (this follows from the formula for the inverse of a matrix, $\left.M^{-1}=\operatorname{det}(M)^{-1} \operatorname{adj}(M)\right)$.

The eigenvalues of a matrix are continuous functions of the matrix entries, see [7]. This means that the smallest and largest eigenvalue, $\lambda_{\min }\left(P_{s}\right)$ and $\lambda_{\max }\left(P_{s}\right)$ respectively, of a symmetric matrix also are continuous functions of the entries of $P_{s}$ (minimum and maximum of a number of continuous functions gives continuous functions).

Using that compositions of continuous functions is continuous we have that $\lambda_{\min }\left(P_{s}\right)$ and $\lambda_{\max }\left(P_{s}\right)$ are continuous functions of $s$.

Now, $\lambda_{\min }\left(P_{s}\right)$ and $\lambda_{\max }\left(P_{s}\right)$ are continuous functions over a closed interval $\Omega$. Hence, they attain finite minimum and maximum values on $\Omega$, i.e., there 
exists $\lambda_{m}<\lambda_{M}<\infty$ such that

$$
\lambda_{\min }\left(P_{s}\right) \geq \lambda_{m} \text { and } \lambda_{\max }\left(P_{s}\right) \leq \lambda_{M} \quad \forall s \in \Omega .
$$

To show the matrix inequalities in the lemma we use the fact that the symmetric matrix $P_{s}$ can be written as $P_{s}=Q_{s}^{T} \Lambda_{s} Q_{s}$, where $Q_{s}^{T} Q_{s}=I$ and $\Lambda_{s}$ is diagonal. This gives

$$
P_{s}-\lambda_{m} I=Q_{s}^{T}\left(\Lambda_{s}-\lambda_{m} I\right) Q_{s} \succeq 0
$$

since $\lambda_{m}$ is less than or equal to all entries of $\Lambda_{s}$. The other inequality is shown similarly and the lemma follows.

Theorem 1 Let $x_{a}$ and $x_{b}$ be two points in $\mathcal{S}_{a s}$ that can be joined by a continuous curve $\Gamma \in \mathcal{S}_{\text {as }}$. Also assume that $\Gamma$ can be chosen to be the projection on $\mathcal{S}_{\text {as }}$ of a continuous curve $\bar{\Gamma} \in \mathcal{E}_{\text {as }}$. Then the state of system (1) can be transferred from $x_{a}$ to a arbitrary neighborhood of $x_{b}$ in a finite amount of time.

Proof: Let the curve $\bar{\Gamma}$ be parameterized as $u=u_{s}=\phi(s)$ and $x=x_{s}=$ $\Phi(s)$ where we can assume, without loss of generality, that $x_{a}=\Phi(0)$ and $x_{b}=\Phi(1)$.

Let $x_{s}$ denote an arbitrarily point on $\Gamma$ and $u_{s}$ the corresponding control (we have $f\left(x_{s}, u_{s}\right)=0$ ). Let $\Gamma_{\epsilon}$ be the set $\Gamma_{\epsilon}=\{x \mid \operatorname{dist}(x, \Gamma) \leq \epsilon\}$ where dist is the Euclidean distance function. Since $f$ is twice continuously differentiable, the linearization of (1) on $\Gamma$ (keeping $u_{s}$ fixed) can be written

$$
\frac{d}{d t}\left(x-x_{s}\right)=A_{s}\left(x-x_{s}\right)+g_{s}(x), \quad A_{s}=f_{x}\left(x_{s}, u_{s}\right)
$$

where $\left|g_{s}(x)\right| \leq K\left|x-x_{s}\right|^{2}, x \in \Gamma_{\epsilon}$ and, $K=\sup _{\operatorname{dist}(x, \Gamma) \leq \epsilon}\left|f_{x x}\left(x, u_{s}\right)\right|$, see [6].

Now, define the following positive definite function

$$
V_{s}(x)=\left(x-x_{s}\right)^{T} P_{s}\left(x-x_{s}\right), P_{s}^{T}=P_{s} \succ 0 .
$$

Using Lemma 1 it is easy to show that $V_{s}(x)$ is a local Lyapunov function for system (1) at $x_{s}$. Here $A_{s}=f_{x}\left(x_{s}, u_{s}\right)=f_{x}(\Phi(s), \phi(s))$ fulfills the conditions of Lemma 1 and $P_{s}$ can be uniformly bounded on $\Gamma$. Furthermore, we have

$$
\begin{aligned}
\dot{V}_{s}(x) & =\left(x-x_{s}\right)\left(A_{s}^{T} P_{s}+P_{s} A_{s}\right)\left(x-x_{s}\right)+2\left(x-x_{s}\right) P_{s} g_{s}(x) \\
& =-\left|x-x_{s}\right|^{2}+2\left(x-x_{s}\right)^{T} P_{s} g_{s}(x) .
\end{aligned}
$$

Here we used (7) to get the third inequality. Now, using the bound on $g_{s}(x)$ from (9) we get

$$
\dot{V}_{s}(x) \leq-\left|x-x_{s}\right|^{2}+2 \lambda_{M} K\left|x-x_{s}\right|^{3}=-\alpha\left|x-x_{s}\right|^{2}
$$


where $\alpha=\left(1-2 \lambda_{M} K\left|x-x_{s}\right|\right)$. From here on we assume that $\left|x-x_{s}\right| \leq r_{1}$, $r_{1}=\min \left(\epsilon, \frac{1}{3 \lambda_{M} K}\right)$, which gives $\alpha \geq \frac{1}{3}$ and $\dot{V}_{s}(x) \leq-\frac{1}{3}\left|x-x_{s}\right|^{2}$.

Now,

$$
\left|x-x_{s}\right|^{2}=\left(x-x_{s}\right)^{T} I\left(x-x_{s}\right) \leq \frac{1}{\lambda_{m}}\left(x-x_{s}\right)^{T} P_{s}\left(x-x_{s}\right)=\frac{1}{\lambda_{m}} V_{s}(x)
$$

which gives

$$
\dot{V}_{s}(x) \leq-\frac{1}{3 \lambda_{m}} V_{s}(x)
$$

Integrating both sides of the inequality between $t_{0}$ and $t$ and using Gronwall's inequality [9] gives the following estimate of the rate of convergence to the state $x_{s} \in \Gamma$

$$
V_{p}(x(t)) \leq V_{p}\left(x\left(t_{0}\right)\right) e^{-\frac{1}{3 \lambda_{m}}\left(t-t_{0}\right)}
$$

which using (12) gives the following estimate

$$
\left|x(t)-x_{p}\right|^{2} \leq \frac{V_{p}\left(x\left(t_{0}\right)\right)}{\lambda_{m}} e^{-\frac{1}{3 \lambda_{m}}\left(t-t_{0}\right)}
$$

Now using the upper bound on the Lyapunov function we finally get the following estimate of the rate of decrease of the distance between $x(t)$ and $x_{s}$

$$
\left|x(t)-x_{s}\right|^{2} \leq\left|x\left(t_{0}\right)-x_{s}\right|^{2} \frac{\lambda_{M}}{\lambda_{m}} e^{-\frac{1}{3 \lambda_{m}}\left(t-t_{0}\right)} .
$$

The condition $\left|x-x_{s}\right| \leq r_{1}$ restricts the region of states for which inequality (15) is valid to $\left|x\left(t_{0}\right)-x_{s}\right|<r_{1} \sqrt{\frac{\lambda_{m}}{\lambda_{M}}}=r \leq r_{1}$. This gives a uniform estimate on both the region of attraction and rate of convergence for points on $\Gamma$.

We can define a finite number,say $N$, of points $x_{1}, \ldots, x_{N}$ on $\Gamma$ in such a way that $\operatorname{dist}\left(x_{j}, x_{j+1}\right)<r / 3, j=1, \ldots, N-1, x_{1}=x_{a}$ and $x_{N}=x_{b}$. Let $u_{j}, j=1, \ldots, N$ be the corresponding control variables on $\bar{\Gamma}$. We now describe how to transfer the state from $B_{r / 3}\left(x_{j}\right)$ to $B_{r / 3}\left(x_{j+1}\right)$. Assuming that the state belongs to $B_{r / 3}\left(x_{j}\right)$, choose the control $u=u_{j+1}$. The state then belongs also to the ball $B_{r}\left(x_{j+1}\right)$. After at most $T=3 \lambda_{m} \log \left(\frac{9 \lambda_{M}}{\lambda_{m}}\right)$ units of time (the time it takes to reduce the radius to $\frac{r}{3}$ ) we know that the state belongs to $B_{r / 3}\left(x_{j+1}\right)$ from the estimate (15). The same procedure can now be used to reach $B_{r / 3}\left(x_{j+2}\right)$ etc. Hence, an estimate of the total time for transferring the state between $x_{a}$ and $B_{r / 3}\left(x_{b}\right)$ is $N T$. Using again the estimate (15) we conclude that the state can come arbitrarily close to $x_{b}$ in a finite amount of time. 
Remark: From the proof it is clear that the state also can be transferred from an initial point in a neighborhood of $x_{a}$ to a neighborhood of $x_{b}$ in finite time.

If the linearization of the system is not asymptotically stable, there is the possibility of active stabilization by state feedback. We first give a technical lemma.

Lemma 2 Let the matrices $A_{s}$ and $B_{s}$ depend continuously on the parameter $s$ which belongs to a closed interval $\Omega$, and assume that $A_{s}, B_{s}$ is stabilizable for all $s \in \Omega$. Also assume that the linear dependence structure of the controllability matrix $\left[B_{s}, A_{s} B_{s}, \ldots, A_{s}^{n-1} B_{s}\right]$ is the same for all $s \in \Omega$ (i.e. if some columns are linearly independent (dependent) for some $s \in \Omega$ they are so for all $s \in \Omega$ ). Then it is possible to choose $L_{s}$ such that

$$
A_{s}-B_{s} L_{s} \text { is Hurwitz, } s \in \Omega
$$

and $L_{s}$ depends continuously on $s$.

Proof: (sketch) Since the dependence structure of the controllability matrix is independent of $s$, it is possible to choose an invertible transformation matrix $T_{s}$ that depends continuously on $s$ such that

$$
T_{s}^{-1} A_{s} T_{s}=\left[\begin{array}{cc}
A_{1, s} & A_{2, s} \\
0 & A_{3, s}
\end{array}\right], \quad T_{s}^{-1} B_{s}=\left[\begin{array}{ll}
B_{1, s} & 0
\end{array}\right]
$$

where the block matrices $A_{1, s}, B_{1, s}$ form a controllable pair and $A_{3, s}$ corresponds to the uncontrollable modes. For a description of this canonical form see e.g. [4]. It follows that $A_{3, s}$ is Hurwitz. By choosing $A_{1, s}, B_{1, s}$ to be in controller form, it is easy to see that the feedback coefficients will depend continuously on $s$ if the desired eigenvalue location for $A_{1, s}$ depends continuously on $s$.

We can now present a strengthened form of Theorem 1.

Theorem 2 Let $x_{a}$ and $x_{b}$ be two points in $\mathcal{S}$ that can be joined by a continuous curve $\Gamma \in \mathcal{S}$. Assume that $\Gamma$ can be chosen to be the projection on $\mathcal{S}$ of a continuous curve $\bar{\Gamma} \in \mathcal{E}$, parameterized by some $s$ belonging to a closed interval $\Omega$. Let the linearization $A_{s}=f_{x}(x(s), u(s)), B_{s}=f_{u}(x(s), u(s))$ satisfy the technical conditions of Lemma 2. Then the state of system (1) can be transferred from $x_{a}$ to an arbitrary neighborhood of $x_{b}$ in a finite amount of time. 
Proof: From Lemma 2 it follows that there exists a continuous feedback matrix $L_{s}$ such that $A_{s}-B_{s} L_{s}$ is Hurwitz. Now the rest of the proof of Theorem 1 applies with $A_{s}$ substituted by $A_{s}-B_{s} L_{s}$ since this matrix satisfies the requirements of Lemma 1. However, the procedure (control law) for transferring the state from $B_{r / 3}\left(x_{j}\right)$ to $B_{r / 3}\left(x_{j+1}\right)$ has to be modified as follows. Assuming that the state belongs to $B_{r / 3}\left(x_{j}\right)$, choose the control $u=u_{j+1}-L_{j+1}\left(x-x_{j+1}\right)$ and we get the same estimate of the finite transfer time between $B_{r / 3}\left(x_{j}\right)$ and $B_{r / 3}\left(x_{j+1}\right)$ as in the proof of Theorem 1 and the theorem follows.

\section{$3 \quad$ Examples}

Example 1 Consider system (6) whose stationary points are shown in Figure 1. It follows immediately from Theorem 1 that it is possible to move between arbitrary states in $(-\infty, 1]$ or in $[-1, \infty)$ by moving along one of the parabolas. Actually one can show that it is possible to move e.g. between $x=-5$ and $x=5$ by applying Theorem 1 twice. The theorem shows that it is possible to move from $x=-5$ to $x=0$ along the right parabola. The control can then be momentarily switched to move to the neighborhood of an equilibrium on the left parabola. Theorem 1 and the remark after our proof of it can then be used to show that the state can be moved along the left parabola to $x=5$.

Example 2 DC-motor.

Consider the following model of a DC-motor

$$
\begin{aligned}
v_{0} & =L \frac{d}{d t} i+R i+K_{1} u \omega \\
J \dot{\omega} & =-B \omega+K_{2} u i
\end{aligned}
$$

where $i$ is the current, the control signal $u$ is the field current, $\omega$ is the angular velocity, and $v_{0}$ the applied voltage. We let the state variables be

$$
x_{1}=i-\frac{v_{0}}{R}, \quad x_{2}=\omega
$$

and rewrite the equations (17) in state space form

$$
\begin{aligned}
& \dot{x}_{1}=-\frac{1}{L} x_{1}-\frac{K_{1}}{L R} u x_{2} \\
& \dot{x}_{2}=-\frac{B}{J} x_{2}+\frac{K_{2}}{J} u x_{1}+\frac{K_{2} v_{0}}{J R} u .
\end{aligned}
$$


The stationary points of (18) are parameterized by $u$ according to

$$
x_{1}=-\frac{K_{1} K_{2} v_{0} u^{2}}{R\left(B R+K_{1} K_{2} u^{2}\right)}, \quad x_{2}=\frac{K_{2} v_{0} u}{B R+K_{1} K_{2} u^{2}},
$$

which corresponds to an ellipse $(-\infty<u<\infty)$ in the state space. Let $u=s$ then the matrix for a linearization of the system is

$$
A_{s}=\left[\begin{array}{cc}
-\frac{1}{L} & -\frac{K_{1} s}{L R} \\
\frac{K_{2} s}{J} & -\frac{B}{J}
\end{array}\right] .
$$

Using the Routh stability criterion we get the following inequalities

$$
\frac{J+B L}{J L}>0, \quad \frac{B R+K_{1} K_{2} s^{2}}{J L R}>0,
$$

which are satisfied for all s since the physical values of the parameters are always positive. Hence, the linearization is globally asymptotically stable for all $s$.

The conditions of Theorem 1 is satisfied for all points on the curve of stationarizable points and hence the state can be transferred between any two points on this curve in finite time (except the point corresponding to $u=s=$ $\pm \infty)$.

Note that the system in this example is globally asymptotically stable for any fixed $u$ since its linearized dynamics (for fixed $u$ ) corresponds to the nonlinear dynamics (the system is bilinear).

Example 3 Cruise control for aircrafts

Consider a very simplified model for velocity control of an aircraft

$$
\begin{aligned}
& \dot{x}_{1}=x_{2}-f\left(x_{1}\right) \\
& \dot{x}_{2}=-x_{2}+u
\end{aligned}
$$

Here $x_{1}$ is the velocity, $x_{2}$ is the engine thrust and $f\left(x_{1}\right)=x_{1}^{2}-2 x_{1}+2$ is the aerodynamic drag. The expression for the drag is typical for some fighter aircrafts operating at high angle of attack and low speed. The mass is normalized to 1 and we have assumed a first order system response from pilot command to engine thrust.

The stationarizable points is given by the parabola

$$
x_{1}^{2}-2 x_{1}+2=x_{2} \text {. }
$$


A linearization of the system around $\left(x_{1}^{0}, x_{2}^{0}\right)$ on the parabola is given by

$$
\Delta \dot{x}=\left[\begin{array}{cc}
2-2 x_{1}^{0} & 1 \\
0 & -1
\end{array}\right] \Delta x+\left[\begin{array}{l}
0 \\
1
\end{array}\right] \Delta u
$$

where $\Delta x=\left(x_{1}-x_{1}^{0}, x_{2}-x_{2}^{0}\right)$ and $\Delta u=u-u_{0}$. We observe that the linearization is asymptotically stable if $x_{1}^{0}>1$. However, since the linearization is controllable (and hence stabilizable) for all points on the parabola we can always find a linear state feedback that stabilizes the linearization around each stationarizable point. According to Theorem 2 it is possible to transfer the state between points on the parabola (20).

Let $u=u_{s}-L\left(x-x_{s}\right)$, where $L=\left[\begin{array}{ll}8 & 2\end{array}\right]$. This expression together with a parameterization of the parabola gives the control law

$$
u=-8 x_{1}-2 x_{2}+6+2 s+3 s^{2},
$$

which can be used to follow the parabola by changing s in small steps, see Figure 2.

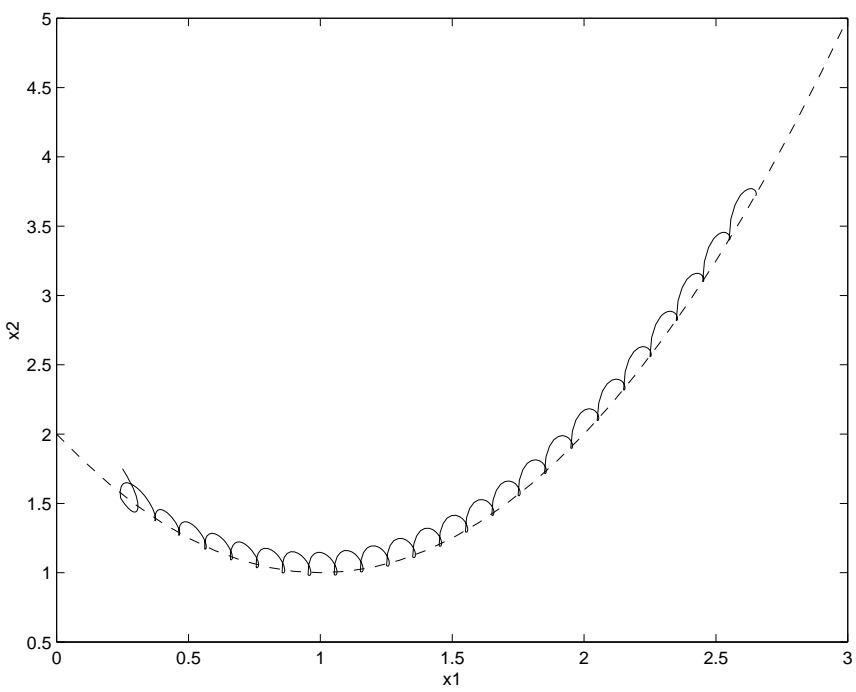

Figure 2: Transfer between equilibrium points of (19). Solid line: trajectory corresponding to small increments of $s$, dashed line: equilibrium points.

Example 4 Consider the following system

$$
\begin{aligned}
& \dot{x}_{1}=-x_{1}^{3}+x_{2} \\
& \dot{x}_{2}=-x_{1}^{2}-x_{2}-x_{2}^{3}+u
\end{aligned}
$$


The stationarizable points is the cubic $x_{2}=x_{1}^{3}$ and the system matrices of a linearization around a point $\left(x_{1}^{0}, x_{2}^{0}\right)$ on this curve is

$$
A=\left[\begin{array}{cc}
-3\left(x_{1}^{0}\right)^{2} & 1 \\
-2 x_{1}^{0} & -1-3\left(x_{2}^{0}\right)^{2}
\end{array}\right], \quad B=\left[\begin{array}{l}
0 \\
1
\end{array}\right]
$$

It can be shown that the linearized system is asymptotically stable for all points on the cubic except for $\alpha<x_{1}^{0}<0$, where $\alpha \approx-0.59$ is a root of a polynomial of degree $\%$. However, the linearized system is controllable everywhere and hence the state can be transferred between any two points on the cubic in finite time according to Theorem 2.

\section{Conclusions}

In this paper we have investigated the problem of moving the state from one equilibrium point to another. We have shown that it is possible to transfer the state between two such points in finite time if they can be joined by a continuous curve of equilibria, provided the linearization at each point is stabilizable.

\section{Acknowledgments}

This work was supported by the Swedish Research Council for Engineering Sciences (TFR), which is gratefully acknowledged.

\section{References}

[1] S. T. Glad and M. Jirstrand. Computational questions of equilibrium calculation with application to nonlinear aircraft dynamics. In Proc. of MTNS'96, St Louis, Missouri, 1996.

[2] J. Hauser. Nonlinear control via uniform system approximation. Systems \& Control Letters, 17:145-154, 1991.

[3] M. Jirstrand. Algebraic Methods for Modeling and Design in Control. Lic. thesis, Dept. of Electrical Engineering, Linköping University, Sweden, 1996.

[4] T. Kailath. Linear systems. Prentice-Hall, Englewood Cliffs, N. J., 1980.

[5] H. Kobayashi and E. Shimemura. Set-point changing for nonlinear systems. International Journal of Control, 50(6):2397-2406, 1989. 
[6] W. Rudin. Principles of Mathematical Analysis. McGraw-Hill, 3rd edition, 1976.

[7] E. D. Sontag. Mathematical Control Theory. Springer-Verlag, 1990.

[8] A. L. Stevens and B. Wigdorowitz. A systematic methodology for the analysis and design of controllers for nonlinear processes. The Transactions of the South African Institute of Electrical Engineers, 3:125-136, 1995 .

[9] F. Verhulst. Nonlinear Differential Equations and Dynamical Systems. Springer-Verlag, 1985. 